\title{
Near-Field Optical Detection of Plasmon Resonance from Gold Nanoparticles: Theoretical and Experimental Evidence
}

\author{
Claudia Triolo • Adriano Cacciola • Rosalba Saija • \\ Sebastiano Trusso • Maria Chiara Spadaro • \\ Fortunato Neri • Paolo Maria Ossi • Salvatore Patanè
}

Received: 30 May 2014 / Accepted: 25 August 2014 / Published online: 26 September 2014

\begin{abstract}
The study of plasmon-induced electromagnetic fields is a very interesting topic for basic research and photonic applications. The plasmon properties depend on many factors, such as composition, size, shape and arrangement of nanoparticles. In this paper, we propose an experimental and theoretical study on the optical properties of gold nanoparticles deposited by pulsed laser ablation and investigated by near-field optical microscopy (SNOM) in a transmission farfield collection scheme. The electromagnetic field properties have been simulated by an exact theoretical analysis based on the multipolar expansion of the fields and on T-matrix approach. The theoretical model almost accurately reproduces the experimental data and makes us confident that the used method is suitable to describe more complex system of metal nanoparticles.
\end{abstract}

Keywords Scanning near-field microscopy $\cdot$ T-matrix · Gold nanoparticle

C. Triolo $\cdot$ A. Cacciola $\cdot$ R. Saija $\cdot$ M. C. Spadaro $\cdot$ F. Neri $\cdot$ S. Patanè Dipartimento di Fisica e di Scienze della Terra, Università di Messina, V.le F. Stagno d'Alcontres 31, 98166 Messina, Italy

S. Trusso $(\triangle)$

CNR-IPCF, Istituto per i Processi Chimico-Fisici, V.le F. Stagno

d'Alcontres 37, 98158 Messina, Italy

e-mail: trusso@me.cnr.it

P. M. Ossi

Dipartimento di Energia \& Centre for Nano Engineered Materials and Surfaces, NEMAS Politecnico di Milano, Via Ponzio, 34-3, 20133 Milan, Italy

Present Address:

M. C. Spadaro

CNR-NANO, Centro di Ricerca S3 and Dipartimento FIM,

Università di Modena e Reggio Emilia,

via G. Campi 213/a, Modena, Italy

\section{Introduction}

It has been shown that the metal nanostructures exhibit very interesting optical properties with respect to their corresponding bulk. Over a wide frequency range, the optical properties of metals can be explained by a plasma model [1]. When the electromagnetic field interacts with a metal, the conduction electrons collectively oscillate in response to the incident field, but their motion is damped by different scattering processes. In order to describe the motion of electron plasma, a damping constant has been defined. This constant is related to the width of the plasmon resonance band and to the lifetime of the electrons scattering. In the bulk metal, the most important contributions for the damping constant are related to the electron-electron and electron-phonon scattering. Instead, the surface effects become important in those nanoparticles with smaller dimensions than the mean free path of electrons [2]. When the wavelength of incident light is much larger than the nanoparticle size, the electronic oscillations are resonant with electromagnetic field. These oscillations are known as surface plasmon resonances (SPRs). In addition to SPRs, the localized surface plasmons (LSPs) may be defined referring to non-propagating excitations of the conduction electrons of metallic nanostructures. If the plasmonic and electromagnetic fields are resonant, an increase of the field located near to the particles can be observed [3]. When two or more plasmonresonant nanoparticles are closely spaced, LSPs of individual particles interact. This new configuration of the electromagnetic field shows additional coupled oscillation modes in gap regions between particles [4]. The resonance condition can be determined from absorption and scattering spectroscopy, and it can be observed that the plasmonic properties of metal nanoparticles are dramatically influenced by their size, shape, composition and arrangement. Moreover, they depend on the medium surrounding the particles and on the characteristics of the incident radiation [5]. 
The study of such plasmon-induced electromagnetic fields plays a fundamental role not only in basic research but also for applications. The SPRs have widely found application in optical communication below the diffraction limit $[1,6,7]$ and in studying those effects that are involved in non-linear surface processes such as surface-enhanced Raman scattering (SERS). Many efforts have been made in order to improve the SERS efficiency of nanostructured substrates. Several fabrication methods, i.e. chemical, physical and electrochemical, have been explored. The main objective was the SERS response improvement through the control of the substrates optical and morphological properties. In the last years, some of us developed a method for the growth of SERS active substrates by pulsed laser ablation in the presence of a controlled gaseous atmosphere [8-10]. Control of the optical and morphological properties was achieved by changing the laser pulse number and the gas ambient pressure while keeping fixed all the other relevant deposition parameters, i.e. laser fluence, gas nature (Ar), target-to-substrate distance and substrate temperature. Controlling these two easily accessible parameters, different surface morphologies can be obtained that lead to different optical properties. A red shift and broadening of the surface plasmon resonance peak are observed moving from a morphology characterized by isolated nanoparticles to percolated structures. In this paper, we report a study of the near-field properties of a gold nanoparticle layer deposited in such a way to obtain a morphology characterized by isolated nanoparticles. The surface number density was large enough to localize the nanoparticles with ease by the SNOM tip and to develop a model to describe the near-field optical response based on the multipolar expansion of the incident, scattered and internal fields. The optical field scattering around a nanoparticle may be viewed as an instantaneous absorption and re-radiation process. When the wavelength of incident light is resonant with plasmon wavelength, the absorption/scattering cross-section of the particle significantly increases. Many SERS studies use gold and silver nanostructures, which have a localized plasmon resonance in the visible or near-infrared spectral region [11-14. Recent studies report possible applications of SPRs in order to enhance the biosensor performances $[15,16]$. Moreover, the use of plasmons for the enhancement of the efficiency and intensity of a conventional LED, as well as the efficiency improvement of thin film solar cells over almost the entire solar spectrum, has been demonstrated $[17,18]$. As a rule, the wavelength of a plasmon mode is smaller than the diffraction limit of light. The scanning near-field optical microscopy (SNOM) achieves a spatial resolution beyond the diffraction limit, and it allows the study of plasmon in metal nanoparticles [19]. The proposed model fully describes the experimental data collected in near field using an aperture SNOM in transmission far-field collection scheme.

\section{Experimental Setup}

\section{Samples}

In previous works [7-9, 20, 21], it was demonstrated that it is possible to grow nanostructured silver and gold thin films by means of pulsed laser ablation (PLA) of solid targets in the presence of a controlled Ar atmosphere. Changing the $\mathrm{Ar}$ pressure and the deposition time while keeping all the other relevant PLA deposition parameters fixed, i.e. laser fluence, substrates temperature and target-to-substrate distance, it is possible to tune the surface morphologies of the thin films. In particular, at low laser shots number, isolated nearly spherical nanoparticles are deposited, while at increasing laser shots number, clustered nanoparticles, islands with smooth edges and, finally, coalesced percolated structures are obtained.

The deposition parameters adopted in this work were chosen on the basis of the previous works $[20,21]$ in order to deposit isolated spherical gold nanoparticles. Gold targets, positioned on a rotating holder in order to avoid excessive surface damage, were ablated using a $\mathrm{KrF}$ excimer laser $(\lambda=$ $248 \mathrm{~nm}$, pulse width $25 \mathrm{~ns}$, repetition rate $10 \mathrm{~Hz}$ ). Glass substrates were positioned in front of the targets at a distance of $35 \mathrm{~mm}$. The samples were grown in the presence of controlled $\mathrm{Ar}$ atmosphere at pressure values of $70 \mathrm{~Pa}$; laser fluence was fixed at $1.8 \mathrm{~J} / \mathrm{cm}^{2}$, and the laser shots number was fixed at 500 in order to avoid nanoparticle coalescence to have still a high number of nanoparticles on the substrates. Images of the sample surface were acquired with a Zeiss Supra 40 field ion scanning electron microscope (SEM). As can be seen in Fig. 1a, the sample morphology is characterized by the presence of isolated nearly spherical gold nanoparticles. From the analysis of size distribution (Fig. 1b), a bimodal size distribution can be observed: nanoparticles with size between 4 and $5 \mathrm{~nm}$ represent the majority of the population besides a fraction of larger nanoparticles having the size of the order of $9 \mathrm{~nm}$. The far-field absorption spectrum (see Fig. 1c), with typical plasmonic peak, was measured by a UV/VIS/ NIR spectrometer (Perkin-Elmer Lambda 2). The green and red lines refer to the exciting sources used in the near-field experiments (see next section) and are reported in comparison to the plasmonic peak position of the investigated sample.

\section{Near-Field Optical Response}

The experiment is sketched in Fig. 2a; it consists of collecting the far-field response of the sample while the distance of a near-field source from the surface changes. To this purpose, we used a tapered optical fibre with a nominal aperture of $50 \mathrm{~nm}$ (Nanonics) installed in a SNOM homemade setup [22]. Two solid-state laser sources were chosen: one at $\lambda=532 \mathrm{~nm}$ 

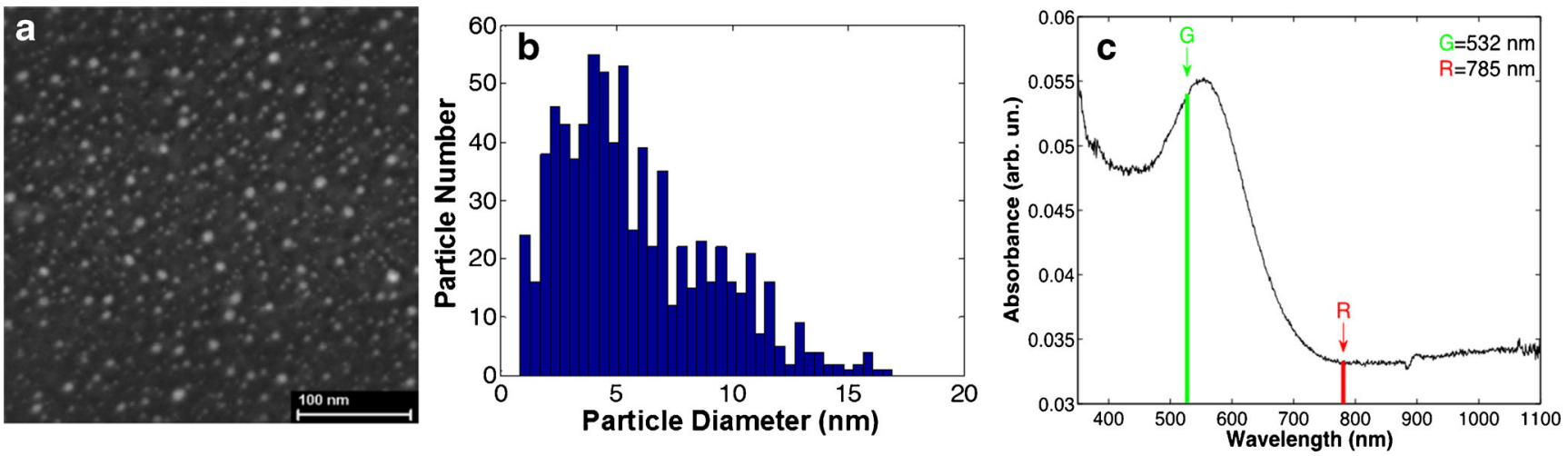

Fig. 1 a SEM image of the surface of the sample deposited at $70 \mathrm{~Pa}$ of Ar with 500 laser shots. b Histogram of particle size distribution. $\mathbf{c}$ Absorption spectrum of Au nanoparticles. The spectral positions of the NSOM excitations are shown with green and red lines

that corresponds to the wavelength of the plasmonic absorption of gold nanoparticles and the second one at $\lambda=785 \mathrm{~nm}$ that is far away from the plasmonic features of the sample. One beam splitter and some mirrors were used to couple the two laser sources to the optical fibre so that it is possible to perform the experiment in the same point of the sample switching on and off one laser or the other. To improve the $\mathrm{S} / \mathrm{N}$ ratio, the lasers were modulated at $2 \mathrm{KHz}$ by an optical chopper (Signal Recovery, Model 197). For a simpler data comparison, a neutral filter with variable density was used to equalize the two laser intensities. The tip-sample distance was controlled by a shear force feedback system based on a tuning fork mechanism $[22,23]$. The optical signal was collected in transmission mode by an objective $20 \times$ installed inside the piezoelectric tube, which works at the same time as a nanoactuator and sample holder. The light collected by the objective was detected by a miniaturized photomultiplier tube (PMT) whose signal was demodulated by a lock-in amplifier (7625 EG\&G Instruments). The optical enhancement as a function of the tip-sample distance was collected during the approach phase, recording at the same time the optical signal and the $z$ height of the piezoelectric tube using first the $532 \mathrm{~nm}$ wavelength. Then, the green laser was switched off, and the experiment was repeated simply switching on the red source and approaching on the same point of the sample.

\section{Results and Discussion}

Figure $2 \mathrm{~b}$ shows the data collected during the tip to the sample surface approaching phase, exciting the sample with the 532 and the $785 \mathrm{~nm}$ wavelengths. The difference of the optical signals acquired using the two wavelengths is noticeable. Using the green light, in fact, the intensity of the optical signals shows a periodic oscillation when the tip-sample distance ranges between a few tens of nanometers, and a couple of microns, besides the signal, shows an enhancement of about $30 \%$ in near field with respect to the far field. At distances below $70 \mathrm{~nm}$, to prevent the tip to crash, the feedback mechanism acts in such a way to stabilize the tip to substrate distance; as a consequence, the optical signal does not change anymore. On the contrary, none of these features occur using the red excitation for which the recorded optical signal is completely flat. The occurrence of the oscillation in the optical signal can be attributed to some resonance effects of the exciting source with the surface plasmon absorption peak. The $532 \mathrm{~nm}$ wavelength, in fact, clearly falls within the plasmonic absorption band, while the $785 \mathrm{~nm}$ excitation wavelength is far away from it, as can be seen in Fig. 1c. The presence of such strong oscillations in the optical signal already during the tip-approaching phase can be of paramount importance when dealing with Raman scattering experiments
Fig. 2 a Scheme of experimental setup for far-field collection system. b Optical data collected in transmission scheme exciting the sample both with the $532 \mathrm{~nm}$ (green line) and $785 \mathrm{~nm}$ (red line) wavelengths
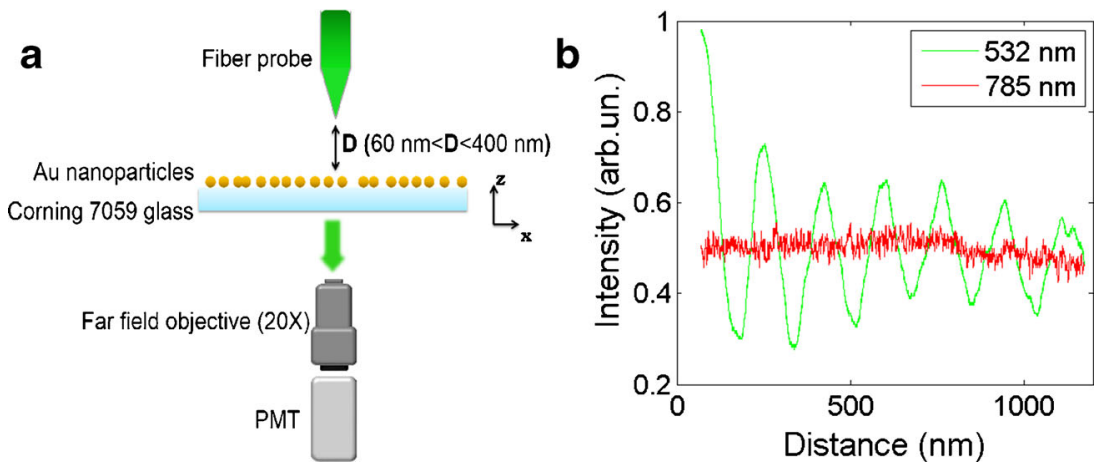
using SNOM setup for achieving spatial resolution below the diffraction limit [NanoRaman]. Despite the relative simplicity of the experiment, a theoretical model describing the interaction of electromagnetic radiation with the metal nanoparticles (MNPs) is needed to relate the observed optical oscillations to the presence of plasmonic resonances [24].

The interaction between the incident and the scattered field in backscattering zone as a function of tip-sample distance can be described resorting to multipolar expansion of electromagnetic fields. Below the methodology used to analyze the measured signal is reported in order to understand at what extent this can give us information on the investigated nanostructures.

Let us start considering a spherical scatterer embedded in a homogeneous medium of (real) refractive index $n$ in which impinges an electromagnetic plane wave with vector propagation $\boldsymbol{k}_{I}=n k_{v} \widehat{\boldsymbol{k}}_{I} \quad$ (with $\left.k_{v}=2 \pi / \lambda\right)$ and polarization direction $\widehat{\boldsymbol{e}}_{I}$ :

$\boldsymbol{E}_{I}=E_{0} \widehat{\boldsymbol{e}}_{I} \exp \left(i \boldsymbol{k}_{I} \cdot \boldsymbol{r}\right)$

and for the scattered field

$\boldsymbol{E}_{S}=E_{0} \frac{e^{\mathrm{ink}_{v} r}}{r} \boldsymbol{f}_{\boldsymbol{I}}\left(\widehat{\boldsymbol{k}}_{S}, \widehat{\boldsymbol{k}}_{I}\right)$

where $\boldsymbol{f}_{\boldsymbol{I}}\left(\widehat{\boldsymbol{k}}_{S}, \widehat{\boldsymbol{k}}_{I}\right)$ is the scattering amplitude whose vector nature gives a correct description of the state of polarization of the scattered wave. For particles of general shape, the scattering amplitude depends both on the direction of propagation of incident field and on the direction of observation $\widehat{\boldsymbol{k}}_{S}$. Furthermore, despite $\boldsymbol{f}_{\boldsymbol{I}}\left(\widehat{\boldsymbol{k}}_{S}, \widehat{\boldsymbol{k}}_{I}\right)$ describes the scattered field in the far zone, its components contain all the information on the near field because they are determined by imposing the boundary conditions across the surface of the particle [24].

A straightforward calculation of the total intensity in the backscattering direction leads us to the following result:

$$
\begin{aligned}
I_{T} & =\left|\boldsymbol{E}_{T}\right|^{2}=\left|\boldsymbol{E}_{S} \cdot \widehat{\boldsymbol{e}}_{I}+\boldsymbol{E}_{I} \cdot \widehat{\boldsymbol{e}}_{I}\right|^{2} \\
& =\left|E_{0} e^{-\mathrm{ink}_{v} \widehat{\boldsymbol{k}} \cdot \boldsymbol{r}}+E_{0} \frac{e^{\mathrm{ink}_{v} r}}{r} \boldsymbol{f}_{I}\left(-\widehat{\boldsymbol{k}}_{I}, \widehat{\boldsymbol{k}}_{I}\right) \cdot \widehat{\boldsymbol{e}}_{I}\right|^{2} \\
& \approx I_{0}\left[1+2 \operatorname{Re}\left(\frac{e^{2 \mathrm{ink}_{v} r}}{r} \boldsymbol{f}_{I}\left(-\widehat{\boldsymbol{k}}_{I}, \widehat{\boldsymbol{k}}_{I}\right) \cdot \widehat{\boldsymbol{e}}_{I}\right)\right]
\end{aligned}
$$

where we have disregarded terms of $1 / r^{2}$ order. Moreover, if we consider that $\boldsymbol{f}_{\boldsymbol{I}} \widehat{\boldsymbol{e}}_{I}$ is in general a complex function of its arguments, setting $\left(\boldsymbol{f}_{\boldsymbol{I}} \cdot \widehat{\boldsymbol{e}}_{I}\right)=\left|\boldsymbol{f}_{\boldsymbol{I}} \cdot \widehat{\boldsymbol{e}}_{I}\right|(\cos \varphi+i \sin \varphi)$, we can rewrite Eq. (3) as follows:

$I_{T} / I_{0} \approx\left[1+\frac{\cos \left(2 \operatorname{ink}_{v} r+\varphi\right)}{r}\left|\boldsymbol{f}_{I}\left(-\widehat{\boldsymbol{k}}_{I}, \widehat{\boldsymbol{k}}_{I}\right) \cdot \widehat{\boldsymbol{e}}_{I}\right|\right]$

At this stage, we can make some considerations. In the backscattering direction, depending on the tip-sample distance $r$, on the incident field, there is superimposed a scattering contribution that (a) harmonically modulates the intensity of the total field, (b) vanishes as $1 / r$, (c) has an intensity that is related to the micro-physical properties of the particle through the scattering amplitude, $\boldsymbol{f}_{\boldsymbol{I}}$.

Regardless of the form and nature of the particle, the modulation due to the interference between the incident and the scattered field in the backward direction has a periodicity given by $T=\lambda /(2 \pi n)$. However, as the optical signal was collected in transmission mode, this periodicity changes since we need to take into account that the total electromagnetic field is recorded after traversing the slab in which the particles are deposited. For this reason, the calculated periodicity matches with the experimental one when we consider $T=\frac{\lambda}{2 \pi n_{1}}$, with $n_{1}=1.55$.

To further analyze Eq. (4), it is necessary to calculate the scattering amplitude and, therefore, to establish the shape, size and number of scatterers that are involved in the experimental measure.

In the following, we will study first what are the information that can be drawn from the experimental data considering that the scatterer is a single gold nanosphere after we will generalize our considerations to more complex situations.

1. The case of single nanosphere

To calculate the scattering amplitude for a single homogeneous nanosphere, we expand the incident plane wave and the scattered field, as well as the field within the sphere, in a series of vector multipole fields. Taking the origin of the coordinates at the centre of the sphere, we have:

$$
\begin{aligned}
& \boldsymbol{E}_{I}=E_{0} \sum_{p l m} \boldsymbol{J}_{l m}^{(p)}(\boldsymbol{r}, k) \boldsymbol{W}_{l m}^{(p)}\left(\hat{\boldsymbol{e}}_{I}, \boldsymbol{k}_{I}\right), \\
& \boldsymbol{E}_{S}=E_{0} \sum_{p l m} A_{l m}^{(p)} \boldsymbol{H}_{l m}^{(p)}\left(\boldsymbol{r}, n k_{v}\right) \\
& \boldsymbol{E}_{I n t}=E_{0} \sum_{p l m} C_{l m}^{(p)} \boldsymbol{J}_{l m}^{(p)}\left(\boldsymbol{r}, n_{0} k_{v}\right)
\end{aligned}
$$

where $n_{0}$ is the refractive (complex) index of the particle, $\boldsymbol{J}_{m}^{(p)}$, defined with the spherical Bessel functions $j_{l}$ and $\boldsymbol{H}_{m}^{(p)}$, defined with the spherical Hankel functions of the first kind $h_{l}$ denotes the multipole fields that satisfy the radiation conditions at origin and at infinity respectively [25]. Imposing the customary boundary conditions at the 
surface of the sphere gets for the amplitudes of the scattered field

$\boldsymbol{A}_{\eta l \mathrm{~m}}^{(1)}=-R_{l}^{(1)} \boldsymbol{W}_{I \eta l m}^{(1)}$ and $\boldsymbol{A}_{\eta l m}^{(2)}=-R_{l}^{(2)} \boldsymbol{W}_{I \eta l m}^{(2)}$

The quantities $R_{l}^{(1)}$ and $R_{l}^{(2)}$ are the well-known Mie scattering coefficients, $b_{l}$ and $a_{l}$, and constitute the elements of the transition matrix for a homogeneous sphere. Now, following the procedure well described elsewhere [24], we can write the scattering amplitude:

$f_{\eta, \eta^{\prime}}=\frac{i}{4 \pi k} \sum_{p l m} \boldsymbol{W}_{S \eta l m}^{(\mathrm{p})} R_{l}^{(\mathrm{p})} \boldsymbol{W}_{I \eta^{\prime} l m}^{(\mathrm{p})}$

that is actually diagonal in $\eta$ on account of the reciprocity theorem [24, 26].

In Fig. 3a, we calculate the quantity $I_{T} / I_{0}$ at $\lambda=532 \mathrm{~nm}$, as a function of the tip-sample distance, varying the observation angle in the vertical plane $x z$. The incidence is along positive $z$-axis, and we start to represent the optical signal at tip-sample distance $>70 \mathrm{~nm}$. The diameter of the gold nanosphere is $d=20 \mathrm{~nm}$ and is embedded
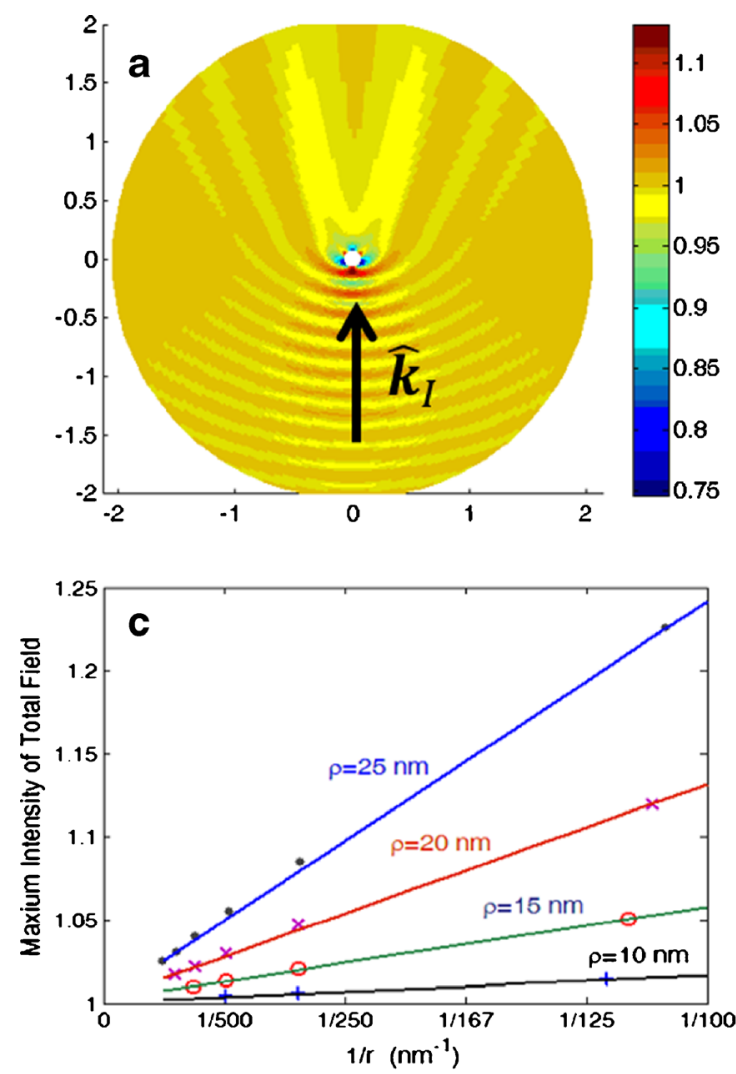

Fig. 3 a Normalized Intensity $\frac{I_{T}}{I_{0}}$ of the total electromagnetic field for a 20 -nm gold sphere. The wavelength of the incident field is $532 \mathrm{~nm}$. The optical signal is computed for a distance tip-sample distance $>70 \mathrm{~nm}$. b Total intensity in the backscattering direction, $-\mathrm{k}_{\mathrm{I}}$, as a function of the distance tip-sample, $r$, for gold nanosphere with different dimensions. c in a medium of refractive index $n=1.55$. The refractive index for the sphere at $\lambda=532 \mathrm{~nm}$ is that reported in the pioneering work by Johnson and Christy [27].

In Fig. 3b, we report $I_{T} / I_{0}$ for the backscattering direction varying the dimension of the nanosphere. It can be observed that the periodicity of the signal is unchanged, as expected, but the intensity of field depends on the size of the particle.

As the signal decreases with a $1 / r$ law, in Fig. 3c, we report, for the gold nanospheres with different size, the intensity of the interference maxima as a function of the inverse of the distance tip-sample, $r$. The best fit of the curves gives us the slopes of the linear functions that are in close relation with the modulus of backscattering amplitude that, for symmetry, are independent on the polarization as $\left|f_{1,1}\left(-\widehat{k}_{I}, \widehat{k}_{I}\right)\right|=\left|f_{2,2}\left(-\widehat{k}_{I}, \widehat{k}_{I}\right)\right|$. At this stage, it is possible to obtain a kind of calibration curve according to which, analyzing the experimental optical signal, is possible to determine the size of the nanoparticle from backscattering. This calibration curve is reported in Fig. 3d, where the modulus of backscattering amplitude results a linear function of $\rho^{2}$.
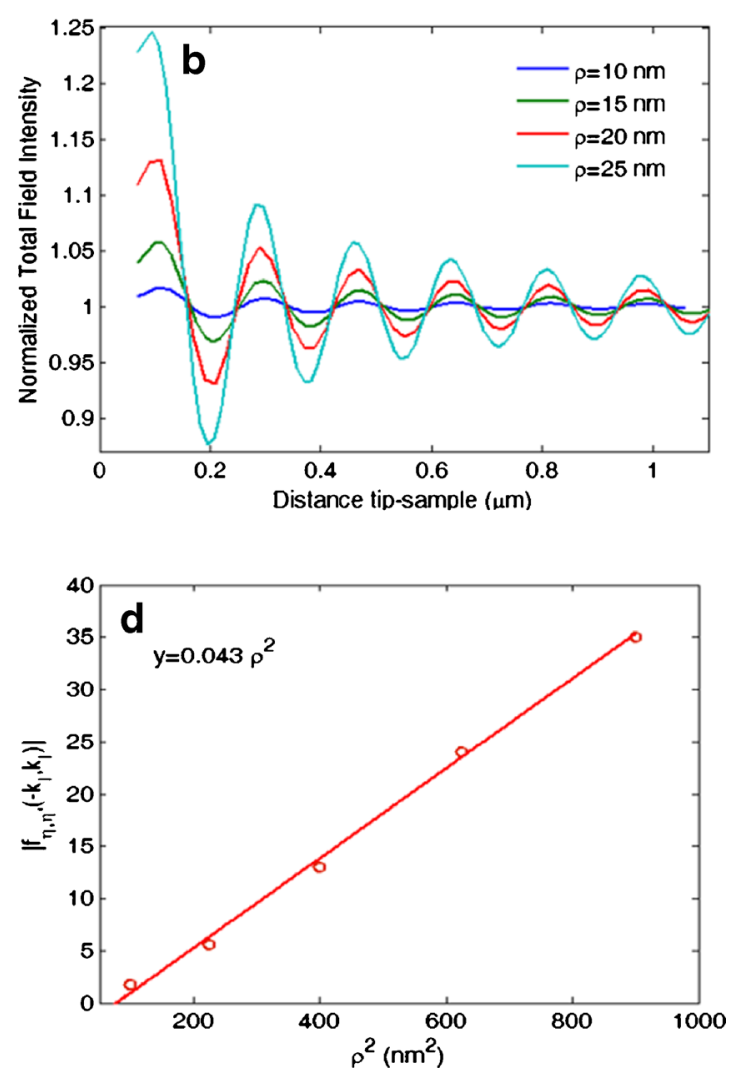

Plot of the maximum of total intensity as a function of $r^{-1}$ for the spheres studied in panel (b). The slopes of the linear fit are related to the modulus of the backscattering amplitudes. d Plot of $\left|f_{\eta, \eta}\left(-\widehat{k}_{I}, \widehat{k}_{I}\right)\right|$ versus $\rho^{2}$ 
2. The case of a cluster of non-touching nanospheres

In this section, we study the total electromagnetic field when the incident field illuminates a surface on which a size distribution of small nanoparticles whose mutual distance may be less than their dimension are deposited. For this reason, resorting to the multipolar expansion of the electromagnetic fields, we calculate the scattering amplitude for a cluster of $N$ non-touching nanospheres [24]. Since the boundary conditions that allow us to determine the scattering amplitude must be imposed at the surface of every sphere of the cluster, we rewrite the incident and the scattered fields in terms of multipole field centred on each sphere:

$\boldsymbol{E}_{l_{l}}=E_{0 \eta} \sum_{p p^{\prime} l l^{\prime} m m^{\prime}} \boldsymbol{J}_{l m}^{(p)}\left(\boldsymbol{r}_{\alpha}, n k_{v}\right) \mathcal{J}_{\text {aoll }}^{\left(p p^{\prime} m m^{\prime}\right.}\left(\boldsymbol{r}_{\alpha}, n k_{v}\right) W_{l \eta^{\prime} m^{\prime}}^{\left(p^{\prime}\right)}$,

$\boldsymbol{E}_{S \eta}=E_{0 \eta} \sum_{p l m}\left[A_{\alpha \eta m}^{(p)} \boldsymbol{H}_{l m}^{(p)}\left(\boldsymbol{r}_{\alpha}, n k_{v}\right)+\sum_{\alpha^{\prime} p^{\prime} l^{\prime} m^{\prime}} \boldsymbol{J}_{l m}^{(p)}\left(\boldsymbol{r}_{\alpha}, n k_{v}\right) \cdot \mathscr{H}_{\alpha \alpha^{\prime} l^{\prime} l^{\prime} m m^{\prime}}^{\left(p p^{\prime}\right)}\left(\boldsymbol{r}_{\alpha}, n k_{v}\right) A_{I l^{\prime} l^{\prime} m^{\prime}}^{\left(p^{\prime}\right)}\right]$

where the scattered field on $\alpha$-th sphere is given by a linear combination of the scattered fields coming from the $N$ - $\alpha$ th's spheres. The $\mathscr{H}$ and $\mathcal{J}$ matrix elements perform the transfer of the multipole fields among different sites. In this way, we obtain for each $\alpha$, a system of linear nonhomogeneous equations, that relates the amplitudes of the
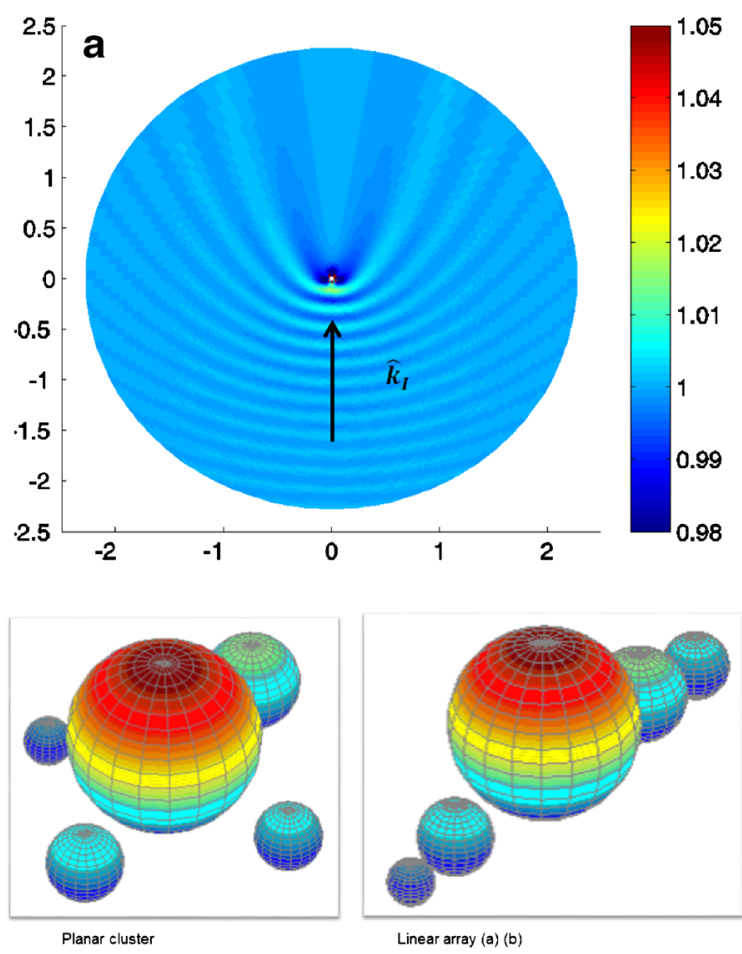

Fig. 4 a Map of the normalized intensity $\frac{\mathrm{I}_{\mathrm{T}}}{\mathrm{I}_{\mathrm{O}}}$ of the total electromagnetic field in the vertical plane $x z$ for a small gold cluster placed on $x y$ plan. The wavelength of the incident field is $532 \mathrm{~nm}$. The optical signal is computed for a distance tip-sample distance $>70 \mathrm{~nm}$. b Optical signal as a function of tip-sample distance in the backscattering direction. The red line is for the linear array; the dashed blue line is for the planar cluster. The dotted black line is for the equivalent nanosphere with $\rho_{\mathrm{eq}}=10.2 \mathrm{~nm}$. c Optical incident field to those of the fields scattered by each sphere in the aggregate.

$A_{\alpha \eta l m}^{(p)}=-\sum_{\alpha^{\prime} p^{\prime} l^{\prime} m^{\prime}} M_{\alpha \alpha^{\prime} l l^{\prime} m m^{\prime}}^{\left(p p^{\prime}\right)} W_{I \eta \alpha^{\prime} l^{\prime} m^{\prime}}^{\left(p^{\prime}\right)}$

In order to define the scattering amplitude for the whole aggregate, it is necessary to refer, once again, the $\alpha$-multicentred fields to the same origin. Finally, we obtain:

$\mathrm{f}_{\eta \eta^{\prime}}=-\frac{\mathrm{i}}{4 \pi k} \sum_{p l m p^{\prime} l^{\prime} m^{\prime}}\left[W_{S \eta l m}^{(p)} \boldsymbol{S}_{l l^{\prime} m m^{\prime}}^{\left(p p^{\prime}\right)} W_{I \eta^{\prime} l^{\prime} m^{\prime}}^{(p)}\right]$

where $\boldsymbol{S}_{l l^{\prime} m m^{\prime}}^{\left(p p^{\prime}\right)}$ are the elements of the transition matrix [24, $28]$ that take into account the morphology of the particle as well as the boundary conditions, but are independent of the state of polarization of the incident field.

In Fig. 4a, we show the map of the total unpolarized field for a small planar cluster of non-touching sphere. The cluster is composed of five nanosphere with radius $\rho_{1}=$ $10 \mathrm{~nm}, \rho_{2}=5 \mathrm{~nm}, \rho_{3}=4 \mathrm{~nm}, \rho_{4}=3.5 \mathrm{~nm}$ and $\rho_{5}=2.5 \mathrm{~nm}$. The particle-particle distance is about $3 \mathrm{~nm}$. In Fig. 4b, we
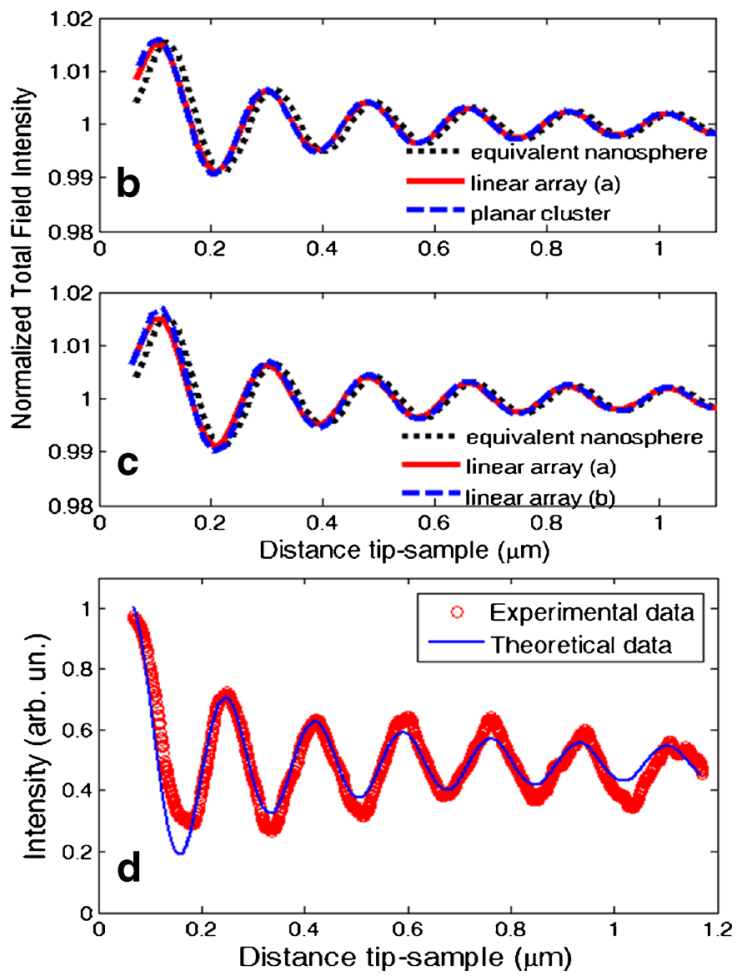

signal as a function of tip-sample distance in the backscattering direction. The red line is for the linear array in which the spheres have $3 \mathrm{~nm}$ of mutual distance; the dashed blue line is for the linear array in which the spheres have $7 \mathrm{~nm}$ of mutual distance. The dotted black line is for the equivalent nanosphere with $\rho_{\mathrm{eq}}=10.2 \mathrm{~nm}$. d Comparison of the experimental optical signal and the theoretical one obtained considering an equivalent gold nanosphere with $\bar{\ell}_{\mathrm{q}}=24.5 \mathrm{~nm}$ 
present the rate $I_{T} / I_{0}$ along the backscattering direction where the optical signal is studied when the geometry of the cluster and the separation among the spheres are changed. For this reason, in panel (b), we varied randomly the spatial arrangement of the spheres going from a cluster with more compact arrangement (planar clusters) to a strongly anisotropic one (linear array). In panel (c), we compare the optical signal for the linear cluster with mutual sphere-sphere distance of 3 and $7 \mathrm{~nm}$. As can be seen, in all cases, the optical backscattering signal is not affected by these changes. This means that each particle can be considered excited only by the external field, and the total scattered field of the medium is the vector sum of the partial scattered fields due to particles. Therefore, as the scattering medium is observed from a distance much greater than its linear dimension and the number of particles is not so large to cause a significant multiplescattering contribution, it is possible to describe the scattering issue in the framework of the single-scattering approximation (SSA) [29]. As a consequence, the scattering amplitude of the whole cluster is given by the sum of the scattering amplitudes of the individual nanoparticles. This can be also interpreted as if the small aggregate, under the analyzed conditions, behaves like an equivalent sphere. The dimension of the equivalent sphere can be determined using the calibration curve presented in Fig. 3d. In panels (b and c), the black dotted curve is for the equivalent sphere whose retrieved radius is $\rho_{\mathrm{eq}}=10.2 \mathrm{~nm}$; the value that is close to the radius of the equivalent volume sphere $\left(\rho_{\mathrm{m}}=10.7 \mathrm{~nm}\right)$ is defined as the sphere that contains the whole mass of the cluster. In addition, we may attribute the slight shift between the equivalent sphere curve and the cluster to the phase factor $\varphi$ that enters in Eq. (4).

Finally, in Fig. 4d, we report the comparison of experimental optical signal and the theoretical one. Analyzing the experimental decrease in signal intensity, for the maxima and minima, the calibration curve gives us for the equivalent sphere the following values: $\rho_{\max }=29.4 \mathrm{~nm}$ and $\rho_{\min }=19.6 \mathrm{~nm}$. In panel (d), we plot the backscattered total intensity for the mean value $\bar{\rho}_{\text {eq }}=24.5 \mathrm{~nm}$.

It is useful to emphasize that the theoretical-experimental agreement is better when the distance tip-sample is still of the order of the wavelength. In this case, in the illuminated area falls a very small number of scatterers. Beyond this distance, when the tip moves away from the surface, the effects due to the presence of any other particle that enters under field illuminated by the SNOM-tip become non-negligible. This causes a not perfect decrease of signal intensity and an increase in the shift between the experimental signal and the theoretical since the number of the illuminated particles increases.
It is interesting to note that the good agreement between the experimental optical signal and the theoretical one is obtained considering an equivalent gold nanosphere of $24.5 \mathrm{~nm}$ in size. Taking into account that the average dimension of the nanoparticles is much smaller (see Fig. 1b), it is quite evident that the detected optical signal comes from a planar cluster of smaller nanoparticles as evidenced by the theory. The observed optical signal intensity decreases,as the distance from the sample surface is increased, which further confirms this hypothesis.

\section{Conclusion}

The understanding of the phenomena of interaction between metal nanoparticles and electromagnetic fields represents a stimulating challenge for researchers in the field of nanooptics. The properties of these nanoparticles, related to the presence of plasmon resonances, appear in their peculiarities when investigated both in the near field and in the far field. In these two regimes, the experimental and theoretical analysis techniques are well established and certified [1]. However, an intermediate regime exists where the electromagnetic far field is influenced by the short range response of each scatterer. In this work, we investigated this regime by transmission SNOM technique, and we described this highly complex system using exact theoretical analysis based on the multipolar expansion of the fields and on T-matrix approach [24, 28]. The only approximation introduced is related to the truncation of multipolar expansions. Despite the small size parameter $x=k \rho \approx 0.2$ of the nanoparticles, which would require multipolar expansion up to $l_{\max } \leq 4$ to obtain an adequate convergence, we use a $l_{\max }=10$ due the critical value of the dielectric constant of gold. The good agreement obtained with the experimental data makes us confident that the model is suitable to describe more complex phenomena due to systems of metal nanoparticles, such as the role of the radial electromagnetic field along the direction of polarization, the enhancement of the scattered field in the near field and the interaction between fields and aggregates.

Acknowledgments R.S. and A.C. acknowledge the helpful discussions with F. Borghese, P. Denti and J.J. Sàenz.

\section{References}

1. Maier SA (2007) Plasmonics: fundamentals and applications. Springer

2. Prasad PN (2004) Nanophotonic. Wiley-Interscience

3. Zayats AV, Smolyanin II, Maradudin AA (2005) Nano-optics of surface plasmon polaritons. Phys Rep 408:131-314 
4. Lin H-Y, Huang C-H, Chang C-H, Lan Y-C, Chui H-C (2010) Direct near-field optical imaging of plasmonic resonances in metal nanoparticle pairs. Opt Express 18:165-173

5. Eustis S, El-Sayed MA (2006) Why gold nanoparticles are more precious than pretty gold: noble metal surface plasmon resonance and its enhancement of the radiative and nonradiative properties of nanocrystals of different shapes. Chem Soc Rev 35:209-217

6. Ridolfo A, Di Stefano O, Fina N, Saija R, Savasta S (2010) Quantum plasmonics with quantum dot-metal nanoparticle molecules: influence of the Fano effect on photon statistics. Phys Rev Lett 105: 263601

7. Savasta S, Saija R, Ridolfo A, Di Stefano O, Denti P, Borghese F (2010) Nanopolaritons: vacuum Rabi splitting with a single quantum dot in the center of a dimer nanoantenna. ACS Nano 4:6369-6376

8. Bailini A, Ossi PM (2007) Expansion of an ablation plume in a buffer gas and cluster growth. Europhys Lett 79:35002

9. Fazio E, Neri F, Ossi PM, Santo N, Trusso S (2009) Growth process of nanostructured silver films pulsed laser ablated in high-pressure inert gas. Appl Surf Sci 255(9676)

10. Fazio E, Neri F, Ossi PM, Santo N, Trusso S (2009) Ag nanocluster synthesis by laser ablation in Ar atmosphere: a plume dynamics analysis. Laser Particle Beams 27:271

11. Agarwal NR, Neri F, Trusso S, Lucotti A, Ossi PM (2012) Au nanoparticle arrays produced by pulsed laser deposition for surface enhanced Raman spectroscopy. Appl Surf Sci 258: 9148-9152

12. Botta R, Upender G, Sathyavathi R, Narayana Rao D, Bansal D (2013) Silver nanoclusters films for single molecule detection using surface enhanced Raman scattering (SERS). Mat Chem Phys 137: 699-703

13. Messina E, Cavallaro E, Cacciola A, Fazio E, D'Andrea C, Gucciardi PG, Iatì MA, Meneghetti M, Compagnini G, Amendola V, Maragò OM (2011) Manipulation and Raman spectroscopy with optically trapped metal nanoparticles obtained by pulsed laser ablation in liquids. J Phys Chem C 115:5115-5122

14. Messina E, Cavallaro E, Cacciola A, Iatì MA, Gucciardi PG, Borghese F, Denti P, Saija R, Compagnini G, Meneghetti M, Amendola V, Maragò OM (2011) Plasmon-enhanced optical trapping of gold nanoaggregates with selected optical properties. ACS Nano 5:905-913

15. Daghestani HN, Day BW(2010) Theory and applications of surface plasmon resonance, resonantmirror, resonant waveguide grating, and dual polarization interferometry biosensors. Sensors 10:9630-9646

16. Li Y, Liu X, Lin Z (2012) Recent developments and applications of surface plasmon resonance biosensors for the detection of mycotoxins in foodstuffs. Food Chem 132:1549-1554

17. Gu X, Qiu T, Zhang W, Chu PK (2011) Light-emitting diodes enhanced by localized surface plasmon resonance. Nanoscale Res Lett 6:199-211

18. Pillai S, Catchpole KR, Trupke T, Green MA (2007) Surface plasmon enhanced silicon solar cells. J Appl Phys 101:093105

19. Billot L, Mortier M, Aigouy L (2013) Near-Field imaging of surface plasmon polaritons excited by chains of gold nanodiscs. Plasmonics $8: 1515$

20. D'Andrea C, Neri F, Ossi PM, Santo N, Trusso S (2009) The controlled pulsed laser deposition of $\mathrm{Ag}$ nanoparticle arrays for surface enhanced Raman scattering. Nanotechnology 20:245606

21. Miotello A, Ossi PM (2009) Laser-surface interactions for new materials production: tailoring structure and properties. Springer

22. Cefalì E, Patanè S, Gucciardi PG, Labardi M, Allegrini M (2007) A versatile multipurpose scanning probe microscope. J Micros 210: 262-268

23. Betzig E, Finn PL, Weiner JS (1992) Combined shear force and nearfield scanning optical microscopy. Appl Phys Lett 60:2484-2486

24. Borghese F, Denti P, Saija R (2007) Scattering from model nonspherical particles. Springer, Berlin Heidelberg

25. Jackson JD (1975) Classical electrodynamics. Wiley, New York

26. Van de Hulst JC (1957) Light scattering by small particles. Wiley, New York

27. Johnson P, Christy R (1972) Optical-constants of noble-metals. Phys Rev B 6:4370

28. Borghese F, Denti P, Saija R (2013) Superposition through phases of the far field scattered by the spheres of an aggregate. J Quant Spectrosc Radiat Transf 129:69-78

29. Mishchenko MI, Hovenier JW, Mackowski DW (2004) Single scattering by small volume element. J Opt Soc Am A 21:71-87 